\section{Una propuesta para mejorar el acceso y cobertura en oncología para Latinoamérica: enfermería de práctica avanzada}

\author{
FRANCISCA MÁRQUEZ-DOREN ${ }^{1, a}$, SILVIA PALMA RIVADENEIRA ${ }^{1-2, b}$, \\ PAZ SOTO FUENTES ${ }^{1-3, c}$, CAMILA LUCCHINI-RAIES ${ }^{1, d}$, \\ JOSÉ PEÑA DURÁN ${ }^{4-5}$, BRUNO NERVI NATTERO ${ }^{2-5}$, \\ PAULINA SUÁREZ PIERART ${ }^{1-2, \mathrm{e}}$, RINA GONZÁLEZ RODRÍGUEZ ${ }^{1, \mathrm{f}}$, \\ NOELIA ROJAS SILVA ${ }^{1, g}$, CLAUDIA BUSTAMANTE TRONCOSO ${ }^{1, \mathrm{~g}}$, \\ CLAUDIA ALCAYAGA ROJAS ${ }^{1, \mathrm{~h}}$, MARÍA ISABEL CATONI SALAMANCA ${ }^{1, \mathrm{i}}$, \\ MARÍA CECILIA ARECHABALA MANTULIZ ${ }^{1, j}$
}

\section{Advanced practice nursing as a proposal to improve access and coverage in oncology for Latin America}

PAHO/WHO proposes to implement the role of Advanced Practice Nurse (APN) in Latin America, to reduce gaps in coverage and access to health care. For this purpose, it is necessary to train nursing professionals with an expanded role, which allows them to collaborate in the diagnosis, treatment, and monitoring of people with specific diseases, under established protocols and clinical guidelines and within consolidated interdisciplinary health teams in a cost-effective way. One of the areas with the greatest coverage deficit in Latin America is adult oncology, with inequality in care opportunities for these patients. Part of the premature deaths attributable to this disease are due to the lack of access to timely diagnosis and treatment. As a contribution to the reduction of this gap, a training program of Advanced Nursing Practice was developed, addressing the most urgent needs in the field of oncology. The necessary competencies were defined to develop a professional-level master's degree program, considering prevention, early detection, diagnosis, treatment, and monitoring of people with oncological diseases at different levels of care. A program of this kind is an opportunity to reduce the access gap and coverage of health care for people with cancer, improving their quality of life and their survival.

(Rev Med Chile 2021; 149: 591-597)

Key words: Advanced Practice Nursing; Education, Graduate; Oncology Nursing.
'Escuela de Enfermería Pontificia

Universidad Católica de Chile.

Centro Colaborador OPS/OMS.

Santiago, Chile.

${ }^{2}$ Centro de Cáncer Red de Salud UC-CHRISTUS. Santiago, Chile.

${ }^{3}$ Asociación Chilena de Educación en Enfermería (ACHIEEN).

Santiago, Chile.

${ }^{4}$ Centro Oncológico, Complejo

Asistencial Dr. Sótero del Río.

Santiago, Chile.

${ }^{5}$ Escuela de Medicina, Pontificia

Universidad Católica de Chile.

Santiago, Chile.

'Enfermera-Matrona, MNSc, PhD.

bEnfermera.

'Enfermera-Matrona, Magíster en

Administración.

'Enfermera-Matrona, MNSc, PhD

eEnfermera, MNSc.

tEnfermera, Magíster en

Sociología.

'Enfermera-Matrona, MNSc.

hEnfermera-Matrona, Magíster en

Psicología de la Salud.

'Enfermera-Matrona, Magíster en

Fundamentación Filosófica.

EEnfermera, Magíster en

Psicología.

Trabajo no recibió

financiamiento.

Los autores declaran no tener

conflictos de interés.

Recibido el 15 de septiembre de 2020, aceptado el 18 de marzo de 2021.

Correspondencia:

Camila Lucchini-Raies

Dirección: Vicuña Mackenna 4860, Macul. Santiago, Chile.

clucchin@uc.cl
L a incorporación de Enfermeras/os de Práctica Avanzada (EPA) ha mostrado internacionalmente, un impacto positivo en resultados clínicos específicos ${ }^{1-6}$, en el acceso a los servicios de salud ${ }^{7,8}$, la satisfacción usuaria ${ }^{5,6,9}$, y en una mejor utilización de los servicios de urgencia, entre $\operatorname{otros}^{2,10}$. El primer programa de EPA se inicia en Estados Unidos en la década de $1960^{11,12}$, después de lo cual se observa un creciente desarrollo y reconocimiento de estos programas. Actualmente, tanto la Organización Panamericana de la Salud (OPS) como la Organización Mundial de la Salud 
(OMS) recomiendan su implementación en el nivel primario de atención, como una estrategia para la promoción de salud y prevención de enfermedades, de manera de aumentar la cobertura y el acceso universal, aportando a mejorar la calidad de atención y resultados sanitarios ${ }^{9,10,13}$.

La EPA es un profesional de enfermería con formación de posgrado (magíster profesional), que ha adquirido conocimientos expertos, habilidades en la toma de decisiones complejas y competencias clínicas que permiten el ejercicio de una práctica extendida ${ }^{9,12}$. Esta última, se entiende como aquella práctica en que la EPA está habilitada para ejercer funciones derivadas del rol médico referidas al diagnóstico y tratamiento de personas con enfermedades agudas o crónicas en condición estable, enmarcadas en un modelo de cuidados de enfermería holístico y centrado en la persona .

OPS/OMS han establecido directrices para la formación de enfermeras/os de práctica avanzada en Latinoamérica y publicado recomendaciones para apoyar su incorporación y fortalecer la resolutividad de los diferentes sistemas de salud. Además, OPS ha concretado reuniones con Ministros de Salud y otros actores relevantes en la toma de decisiones en Latinoamérica, para incorporar la EPA en sus planes de trabajo ${ }^{10}$; con resultados disímiles en la región. En Chile, desde el ámbito académico y clínico, se ha reflexionado y avanzado en la creación de propuestas para su implementación, que se encuentran en desarrollo ${ }^{12}$.

Abordar la cobertura y el acceso universal a la salud implica asegurar la disponibilidad de servicios de salud integrales y de calidad, a lo largo del ciclo vital y el acceso equitativo, independientemente de las condiciones sociales ${ }^{14}$. Chile cuenta con un sistema de salud mixto, público/privado, que garantiza el acceso universal y la calidad del tratamiento para 85 condiciones explícitamente definidas, 19 se relacionan directamente con cáncer ${ }^{15}$. Se estima que se han beneficiado con esta política de salud las personas más desfavorecidas del país ${ }^{16}$.

No obstante los logros antes descritos, las inequidades aún persisten, por lo que se requiere seguir avanzando en el acceso a la salud, en especial para algunos grupos vulnerables como las personas mayores, en situación de pobreza y en comunidades rurales. Asimismo, se requiere incrementar los servicios de promoción, prevención y gestión de la salud para reducir la carga y mortalidad asociada a enfermedades crónicas, especialmente en salud mental, cáncer, enfermedades cardiovasculares y diabetes ${ }^{17}$.

Dado que el cáncer constituye la segunda causa de muerte en Chile $^{18}$ y es una de las tres primeras causas de mortalidad en los países de Latinoamérica y el Caribe ${ }^{19}$, el propósito de este artículo es presentar una propuesta para implementar el rol de EPA en Latinoamérica, dirigida a la atención de personas con problemas oncológicos, como una forma de avanzar en los lineamientos de OPS/ OMS respecto de la mejora en el acceso y cobertura universal en salud ${ }^{9}$.

\section{¿Por qué se necesita una EPA en oncología?}

Latinoamérica ha vivido una transición demográfica y epidemiológica en las útlimas décadas, influída por cambios en los estilos de vida, factores ambientales, conductuales y económicos. Lo anterior ha llevado a que las enfermedades no transmisibles reemplacen o coexistan con aquellas transmisibles, lo que explica las tendencias de mortalidad en los distintos países de la región ${ }^{19}$.

Chile cuenta con buenos indicadores de salud pública, entre los que destacan la reducida tasa de mortalidad infantil, la alta expectativa de vida al nacer, la alta cobertura de atención profesional del parto y de inmunizaciones, entre otros ${ }^{20}$. Sin embargo, surge el desafío del envejecimiento creciente de la población, una alta prevalencia de enfermedades crónicas no transmisibles, como el cáncer, la alta carga de factores de riesgo en población adulta e infantil y la baja proporción de factores protectores de la salud ${ }^{20}$.

También persisten brechas de acceso y cobertura en la atención en áreas con alto impacto en salud. En Chile, como en otros países de la región, la relación de profesionales médicos y enfermeras por cada mil habitantes es menor a la recomendación de la Organización para la Cooperación y Desarrollo Económico (OCDE), siendo esta situación aún más desfavorable en sectores aislados y de difícil acceso ${ }^{21}$.

Una de las áreas críticas en cuanto a acceso y cobertura es la atención oncológica, debido principalmente a los prolongados tiempos de latencia para la primera atención, que resultan clave para el pronóstico del paciente y el costo en salud; al fallecimiento de personas que mueren esperando 
atención; y la falta de médicos especialistas en oncología 22 .

En el caso de Chile, de acuerdo al registro de la Superintendencia de Salud, se cuenta con 0,5 médicos hemato-oncólogos por cada 100.000 habitantes y alrededor de 158 en todo el país, estimándose que la necesidad nacional actual es de 400 especialistas $^{16,23}$. Existen solo cuatro programas acreditados de formación para oncólogos en el país, de los que egresan, 10 a 12 profesionales cada año. De igual manera, hay un gran déficit de otros especialistas para la atención de pacientes con cáncer (radioterapeutas, hematólogos, cirujanos oncólogos, entre otros); asimismo, existe una falencia de especialistas de otras profesiones de la salud con las competencias necesarias para la atención de pacientes oncológicos, como enfermeras, kinesiólogos, nutricionistas, psicólogos, terapeutas ocupacionales, entre otros. Lo anterior, deja en evidencia la crítica situación de cobertura y acceso para las personas con cáncer a nivel nacional; situación que puede ser extrapolable a otros países de la región.

Dada la problemática actual es que se requiere contar con profesionales competentes y calificados, que lleven a cabo acciones de promoción, prevención, diagnóstico, tratamiento y seguimiento de personas con cáncer y sus familias, mejorando la resolutividad en el contexto de un equipo oncológico interprofesional consolidado, que permitan dar respuesta a las necesidades actuales de la población ${ }^{23}$.

Si bien, tradicionalmente se ha considerado al médico como el recurso profesional para la resolución de los problemas oncológicos, la presente propuesta plantea ampliar el horizonte al aporte del profesional EPA en oncología para la resolución de estos problemas, considerando que se trata de un profesional preparado para responder a las necedidades de salud de las personas con cáncer y sus familias ${ }^{24}$. Actualmente, en Chile se cuenta con un grupo de más de setecientos profesionales de enfermería trabajando en el área oncológica, según el capítulo de enfermería de la Sociedad Chilena de Oncología, quienes podrían ser los primeros en acceder a la formación entregada por este programa, lo que supone una oportundiad para contar con profesionales altamente capacitados en un período acotado de tiempo. Lo anterior se fundamenta en la sólida formación del profesional de enfermería, que se caracteriza por la adquisición de competencias en las áreas clínica, de gestión, educación e investigación, que le permiten tener una visión integral y comprensiva de las personas y familias a su cuidado; junto con su experiencia clínica al cuidado de pacientes oncológicos. Este punto es clave porque se trata de profesionales cuya formación requiere de una menor inversión económica y de tiempo, si se compara con la requerida para la formación de especialistas médicos, lo que permite pensar en la factibilidad de esta estrategia ${ }^{25}$.

\section{¿Qué EPA en oncología se propone formar?}

En la región, existe una amplia formación de enfermeras/os generalistas a nivel de pregrado; sin embargo, la formación de posgrado conducente a especialidades clínicas, magíster y doctorado es menor. A nivel nacional, solo dos escuelas de enfermería cuentan con programas de especialización en el área oncológica, de los cuales entre el 2010 y 2018 egresaron 149 especialistas. Cabe destacar que en el país trabajan un total de 757 enfermeras/os en servicios relacionados con oncología (públicos y privados) y de ellos solo el $15 \%$ es especialista ${ }^{26}$.

Sin embargo, el contar con enfermeras/os especialistas en oncología, no soluciona el problema de acceso y cobertura en la atención. Lo anterior es esperable, dado que su rol no contempla competencias ampliadas. Para que las/os enfermeras/os puedan adquirir dichas competencias, se requiere cubrir una brecha en áreas específicas que incluyen la formación avanzada en valoración física, farmacología y fisiopatología, así como también experiencias clínicas que le permitan ejercer este $\mathrm{rol}^{7}$.

Se propone formar una EPA visionaria, autónoma, proactiva, innovadora, capaz de comprender y abordar las necesidades y problemas derivados del continuo salud-enfermedad de personas con problemas oncológicos y sus familias, que profundice en la adquisición de competencias para una toma de decisiones más complejas, basadas en un alto grado de conocimiento y experticia clínica oncológica. Se espera que el foco de su práctica, que se lleva a cabo en colaboración interdisciplinaria, esté centrada en la persona y familia; asumiendo un rol extendido, innovador y de consultoría, facilitador y motivador de equipos; en un contexto cambiante, a fin de promover, mejorar 
y recuperar la salud de las personas a través de un cuidado integral, progresivo, seguro, resolutivo y de calidad. Para lograr lo anterior se siguió el modelo propuesto por Hamric y colaboradores ${ }^{7}$, que establecen siete competencias clave a desarrollar por una EPA, las que se definen en la Tabla 1.

\section{¿Cuál ha sido la metodología de trabajo para implementar el programa de formación propuesto?}

La Escuela de Enfermería UC en conjunto con médicos y enfermeras del Centro de Cáncer de la Red de Salud UC-CHRISTUS, han propuesto desarrollar un programa de posgrado para la formación de EPA, que permita abordar las necesidades de salud más urgentes en el ámbito de la oncología.

En base a las siete competencias ${ }^{7}$, se llevó a cabo un levantamiento para el área oncológica, considerando a los sectores de salud público y privado, con el objetivo de identificar aquellas competencias adquiridas y determinar la brecha de formación existente entre enfermeras/os especialistas en el área y la EPA que se espera formar. Como resultado, de las siete competencias establecidas, la de cuidado clínico experto era en la que existía la brecha más importante. Esto concuerda con el desempeño de la EPA, puesto que es en esta competencia donde se adquieren las habilidades para desarrollar el rol ampliado (diagnóstico, tratamiento y seguimiento).

Para abordar la brecha identificada, se elaboraron sub-competencias de la competencia de cui-

\section{Tabla 1. Definición de las siete competencias del perfil de egreso de la Enfermera} de Práctica Avanzada en Oncología

\begin{tabular}{|c|c|}
\hline Competencia & Definición de competencia \\
\hline $\begin{array}{l}\text { Cuidado clínico } \\
\text { experto }\end{array}$ & $\begin{array}{l}\text { Establece una relación terapéutica con el paciente y su familia, en donde se busca una comprensión } \\
\text { mutua de los problemas de salud a través de la recogida de información y una comunicación efectiva. } \\
\text { Evalúa opciones de manejo y tratamiento, lleva a cabo acciones de diagnóstico, seguimiento, trata- } \\
\text { miento farmacológico y no farmacológico bajo protocolos clínicos; ejecuta acciones educativas de } \\
\text { apoyo, de consejería y confort y; por último, participa en la toma de decisiones en relación a acciones } \\
\text { futuras. La farmacología, la fisiopatología y la valoración avanzadas, respaldan esta competencia. }\end{array}$ \\
\hline $\begin{array}{l}\text { Relación terapéutica } \\
\text { y abogacía } \\
\text { (defensoría) }\end{array}$ & $\begin{array}{l}\text { Promueve el automanejo de enfermedades crónicas, realiza acompañamiento a los pacientes durante } \\
\text { el proceso de transición salud-enfermedad, aplica el modelo transteórico del cambio de conducta, } \\
\text { desarrolla habilidades para la resolución y negociación frente al conflicto, y realiza acompañamiento } \\
\text { de pacientes complejos y sus familias. Además, ejerce el rol de abogacía, con una visión global y } \\
\text { de solidaridad. }\end{array}$ \\
\hline Mentoría/consultoría & $\begin{array}{l}\text { Ejerce un rol de mentoría a enfermeras/os en formación y de consultoría a pares y/o al equipo de } \\
\text { salud. Hace la diferencia entre la consultoría, que puede ser solicitada para apoyar la resolución de } \\
\text { casos por otras EPA, y la colaboración para el cuidado de personas y sus familias. }\end{array}$ \\
\hline $\begin{array}{l}\text { Práctica basada en } \\
\text { evidencia }\end{array}$ & $\begin{array}{l}\text { Utiliza el proceso de práctica basada en evidencia en el que incorpora su expertiz clínica, la evidencia } \\
\text { científica disponible y los valores y preferencias del paciente y su familia, para la toma de decisiones } \\
\text { clínicas y la creación de cambios significativos en la práctica. }\end{array}$ \\
\hline Liderazgo & $\begin{array}{l}\text { Ejerce el liderazgo clínico y profesional, caracterizado por propiciar el empoderamiento de otros, la } \\
\text { innovación, el involucramiento político, y ser agente de cambio y emprendimiento; abarcando las } \\
\text { cuatro funciones del rol, a nivel de sistema y políticas de salud. }\end{array}$ \\
\hline $\begin{array}{l}\text { Trabajo } \\
\text { interprofesional } \\
\text { (colaboración) }\end{array}$ & $\begin{array}{l}\text { Ejerce su rol en colaboración con otros profesionales, basado en un modelo de atención de salud } \\
\text { caracterizado por la comunicación efectiva, el respeto y confianza mutua. Realiza acciones que le } \\
\text { permiten liderar la transición del cuidado del paciente y su familia entre los diferentes escenarios } \\
\text { de atención clínica. }\end{array}$ \\
\hline $\begin{array}{l}\text { Toma de decisiones } \\
\text { éticas }\end{array}$ & $\begin{array}{l}\text { Considera en su actuar los principales dilemas éticos en enfermería tales como, problemas de comu- } \\
\text { nicación, conflicto interdisciplinario y compromisos múltiples. Así como también, las dimensiones } \\
\text { éticas del cuidado que afectan la práctica avanzada como las condiciones de salud agudas y crónicas, } \\
\text { aspectos sociales y acceso a recursos de las personas a su cuidado. Además, considera aspectos } \\
\text { legales del ejercicio del rol. }\end{array}$ \\
\hline
\end{tabular}


dado clínico experto, específicas para oncología. Esta construcción contó con la participación de enfermeras clínicas y académicas del área oncológica y médicos oncólogos, con el fin de validar las competencias planteadas y al mismo tiempo establecer la factibilidad de llevar a cabo dicho rol.

Estas sub-competencias clínicas corresponden al ámbito de acción de la EPA en oncología en su rol ampliado, en los tres niveles de atención, abarcando acciones de prevención y detección en el nivel primario; de valoración específica, tratamiento y cuidado avanzado, y de seguimiento, en los niveles secundario y terciario. De esta manera, se estableció que la EPA en oncología utiliza herramientas y conocimientos avanzados basados en evidencia, para proveer cuidados a personas con cáncer, a los familiares en su rol de cuidadores, y promover las mejores prácticas en la prevención, detección y tratamiento del cáncer. En la Tabla 2 se presentan los niveles de atención, las acciones específicas y las subcompetencias del área clínica, que requiere desarrollar la EPA en Oncología.

En base a lo anterior, se propuso un plan específico para incorporar la EPA, primariamente en Chile, con proyecciones a expandir la formación a otros países de la región. Lo anterior, implica un cambio que tiene múltiples alcances, como son la definición y validación de un nuevo rol profesional para enfermería, la definición de aspectos de financiamiento de la EPA en oncología en los sistemas de salud, implicancias legales, entre otros.

Para contribuir a la solidez del programa de estudios de EPA, se contemplan cursos de profundización en ciencias básicas que respondan a los requerimientos definidos en las competencias y subcompetencias descritas anteriormente, prácticas clínicas de $1.300 \mathrm{~h}$ que consideran estadías en servicios de la especialidad y un perfil de egreso validado a nivel nacional e internacional por académicos con experiencia en formación de EPA, por enfermeras/os clínicas/os con experiencia en ejercicio de este rol y por empleadores. El proceso de validación por parte de los actores clave y particularmente por parte de los empleadores, es especialmente importante para introducir este nuevo rol profesional con pertinencia contextual.

Para avanzar en este proceso la Escuela de Enfermería UC y el Centro de Cáncer de la Red de Salud UC-CHRISTUS, implementaron un programa piloto cuya primera etapa consistió en formar a un grupo de enfermeras del área oncológica que ya desempeñaban parte del rol extendido. A la fecha, estas profesionales han completado la primera etapa de formación teórica a través de cuatro cursos que forman parte central del programa: "Rol de las Enfermeras de Práctica Avanzada", "Farmacología Clínica en Enfermería de Práctica Avanzada", "Fisiopatología Avanzada" y "Valoración Integral de Salud Avanzada". Estos cursos les han permitido profundizar en contenidos teóricos para el desempeño del nuevo rol, y posteriormente en una segunda etapa, bajo supervisión médica especializada, comenzar a adquirir experiencia clínica en el ejercicio del mismo.

Tabla 2. Subcompetencias clínicas del rol ampliado según acción específica y nivel de atención de salud

\begin{tabular}{|c|c|c|c|c|}
\hline \multirow[t]{2}{*}{$\begin{array}{l}\text { Nivel de } \\
\text { atención }\end{array}$} & $\begin{array}{l}\text { Prevención y } \\
\text { detección }\end{array}$ & $\begin{array}{c}\text { Valoración específica } \\
\text { avanzada }\end{array}$ & $\begin{array}{c}\text { Tratamiento y } \\
\text { cuidado }\end{array}$ & Seguimiento \\
\hline & \multicolumn{4}{|c|}{ Acción específica } \\
\hline $\begin{array}{l}\text { Nivel Atención } \\
\text { Primario }\end{array}$ & $\begin{array}{c}\text { Demuestra conocimien- } \\
\text { to experto en oncología } \\
\text { para la prevención } \\
\text { y detección de estas } \\
\text { patologías }\end{array}$ & & & $\begin{array}{l}\text { Seguimiento en perso- } \\
\text { nas con cáncer tratados } \\
\text { con intención curativa } \\
\text { o paliativa a largo plazo } \\
\text { (más de } 5 \text { años) }\end{array}$ \\
\hline $\begin{array}{l}\text { Nivel Atención } \\
\text { Secundario y } \\
\text { Terciario }\end{array}$ & & $\begin{array}{l}\text { Valoración avanzada } \\
\text { para el diagnóstico de } \\
\text { cáncer en el adulto y de } \\
\text { condiciones de salud } \\
\text { asociadas }\end{array}$ & $\begin{array}{c}\text { Tratamiento y cuidado } \\
\text { avanzado en personas } \\
\text { con diagnóstico esta- } \\
\text { blecido por oncología } \\
\text { médica una vez finali- } \\
\text { zado el primer ciclo de } \\
\text { quimioterapia }\end{array}$ & $\begin{array}{l}\text { Seguimiento en } \\
\text { personas con cáncer } \\
\text { tratados con intención } \\
\text { curativa o paliativa a } \\
\text { corto plazo en atención } \\
\text { secundaria y terciaria }\end{array}$ \\
\hline
\end{tabular}


Para favorecer la implementación de este nuevo rol profesional, se requiere del apoyo tanto de las autoridades académicas y sanitarias, como del desarrollo de una estrategia específica para su implementación, que considere los facilitadores y obstaculizadores para el cambio. Para ello, se propone la formación de una masa crítica de EPAs que puedan instalar el nuevo rol y formar a las futuras generaciones; la habilitación de modelos clínicos para el ejercicio de este rol; y la creación de un programa académico conducente al grado de Magíster. Para llevar a cabo estas acciones, se requiere el trabajo colaborativo entre representantes de la academia, de los gremios de enfermería y medicina, de los ministerios de salud y de las entidades encargadas de velar por la calidad de la formación de profesionales de enfermería a nivel local ${ }^{1}$.

\section{Conclusiones}

La implementación de EPA en oncología, se presenta como una oportunidad para aportar a la solución de los problemas de acceso y cobertura en salud de personas con cáncer, a un costo abordable a la realidad del contexto latinoamericano. Los programas académicos para la formación de EPA en oncología deben considerar al menos siete competencias, siendo central el cuidado clínico directo experto avanzado en las áreas de valoración para la detección, tratamiento y cuidado en personas con diagnóstico de cáncer, y seguimiento en pacientes tratados con intención curativa o paliativa.

La creación de un programa académico de formación de EPA en Latinoamérica enfrenta desafíos en la capacitación de recurso humano y en la implementación del rol. Se recomienda que la implementación de este nuevo rol se realice inicialmente en áreas donde el equipo interprofesional esté de acuerdo, las/os enfermeras/os estén desarrollando algunas funciones del rol extendido, los médicos estén dispuestos colaborar activamente en la formación y asumir la supervisión de este rol, y se cuente con un programa académico sólido para su formación.

Agradecimientos: Los autores agradecen el apoyo brindado para el desarrollo del programa académico de EPA a Susan Kelly-Weeder, PhD,
Associate Dean for Graduate Programs and Associate Professor at the Connell School of Nursing, Boston College. Además, agradecen a la Facultad de Medicina de la Pontificia Universidad Católica de Chile, a la Red de Salud UC-CHRISTUS, a la Fundación Chile Sin Cáncer, y a la Dirección de Asuntos Internacionales de la Escuela de Enfermería UC, por apoyar la creación de este programa académico.

\section{Referencias}

1. Bryant-Lukosius D, DiCenso A. A framework for the introduction and evaluation of advanced practice nursing roles. Nurs Heal Care Manag Policy 2004; 48 (5): 530-40.

2. Brown S, Grimes D. A meta-analysis of nurse practitioners and nurse midwives in primary care. Nurs Res. 1995; 44 (6): 332-9.

3. Bodenheimer T, MacGregor K, Stothart N. Nurses as leaders in chronic care. Their role is pivotal in improving care for chronic diseases. BMJ. 2005; 330: 612-3.

4. Riviotta A, Galiano A. Enfermeras de Práctica Avanzada para Mejorar los Resultados de los Pacientes Hospitalizados: Una Revisión Sistemática. Rev Iberoam Educ e Investig en Enfermería 2018; 8 (1): 48-61.

5. Kennedy F, Mcdonnell A, Gerrish K, Howarth A, Pollard C, Redman J. Evaluation of the impact of nurse consultant roles in the United Kingdom: A mixed method systematic literature review. J Adv Nurs. 2012; 68 (4): 721-42.

6. Donald F, Martin-Misener R, Carter N, Donald EE, Kaasalainen S, Wickson-Griffiths A, et al. A systematic review of the effectiveness of advanced practice nurses in long-term care. J Adv Nurs. 2013; 69 (10): 2148-61.

7. Hamric A, Hanson C, Tracy MF, O Grady E. Advance Practice Nursign. An Integraive Approach. 5th ed. Hamric A, Hanson C, Tracy MF, O`Grady E, editors. St. Luise: Sounders - Elsevier 2014; 752.

8. Newhouse RP, Stanik-hutt J, White KM, Johantgen M, Bass EB, Zangaro G, et al. 1990-2008: A Systematic Review. Nurs Econ [Internet]. 2011; 29 (5): 1-22. Available from: https://www.nursingeconomics.net/ce/2013/ article3001021.pdf

9. Organización Panamericana de la Salud OPS. Ampliación del rol de las enfermeras y enfermeros en la atención primaria de salud [Internet]. 2018. Available from: http://iris.paho.org/xmlui/bitstream/handle/123456789/34959/9789275320037_spa.pdf?sequence $=5$ \&isAllowed $=y$. 
10. Cassiani SHDB, Zug KE. Promoting the Advanced Nursing Practice role in Latin America. Rev Bras Enferm [Internet]. 2014; 67 (5): 673-4. Available from: http://www.scielo.br/scielo.php?script=sci_arttext\&pi$\mathrm{d}=$ S0034-71672014000500673\&lng=en\&nrm=iso\&tln$\mathrm{g}=$ en\%0Ahttp://www.scielo.br/pdf/reben/v67n5/00347167-reben-67-05-0677.pdf.

11. AACN. The Essentials of Master's Education for Advance Practice Nursing. Education. 2006.

12. Aguirre-Boza F. Enfermería de Práctica Avanzada en la atención primaria: Ahora es el momento. Puntos Ref Cent Estud Públicos [Internet]. 2016; 447 (Noviembre 2016): 1-16. Available from: www.cepchile.cl

13. Cassiani SHDB, Rosales LK. Initiatives towards Advanced Practice Nursing Implementation in the Region of the Americas. Esc Anna Nery - Rev Enferm [Internet]. 2016; 20 (4): 4-5. Available from: http://www.gnresearch.org/doi/10.5935/1414-8145.20160081.

14. OPS/OMS. Acceso universal a la salud y cobertura universal de salud: cumbre enfermería de práctica avanzada 2015.

15. Becerril-Montekio V, Reyes J de D, Annick M. Sistema de salud de Chile. Salud Publica Mex. 2011; 53 (2): s13243.

16. MINSAL. Superintendencia de Salud. Patologías garantizadas AUGE.

17. Ministerio de Salud de Chile. Encuesta Nacional de Salud 2016-2017 Primeros resultados [Internet]. Departamento de Epidemiología, División de Planificación Sanitaria, Subsecretaría de Salud Pública. 2017. Available from: http://web.minsal.cl/wp-content/ uploads/2017/11/ENS-2016-17_PRIMEROS-RESUL-
TADOS.pdf.

18. DEIS/MINSAL. Serie de defunsiones 2000-2016 [Internet]. Defunsiones por alguna causa específica según sexo 2017. Available from: http://www.deis.cl/ wp-content/2017/gobCL-sitios-1.0/assets/SerieDefunciones_2000_2015.html.

19. OMS, OPS. Salud Américas. Resumen: panorama regional y perfiles de país 2017.

20. Organización Panamericana de la Salud OPS. Salud en Chile 2010.

21. OECD. Health at a Glance 2017: OECD Indicators. OECD Publishing. 2017; 216.

22. Comisión Asesora MINSAL. Estado de situación personas fallecidas en listas de espera NO GES y garantías retrasadas GES [Internet]. Informe Comisión Médica Asesora Ministerial. Santiago 2017. Available from: http://www.senado.cl/site/presupuesto/2017/ cumplimiento/Glosas 2017/tercera_subcomision/16 Salud/3412Salud/Informe Final Comision Asesora LE y Garantias Retrasadas GES 17082017.pdf.

23. Ministerio de Salud de Chile. Plan Nacional de Cáncer 2018 - 2028 [Internet]. Santiago 2018. Available from: https://cdn.digital.gob.cl/filer_public/d3/0a/d30a1f5e53d9-4a31-a4fe-e90d8d9a2348/documento_plan_nacional_de_cancer.pdf.

24. Gaviria-Noreña DL. Planning the required nursing personnel to respond to care needs. Investig y Educ en Enferm. 2013; 31 (1): 116-24.

25. Schwarting I. Position paper. Proc New Secur Paradig Work. 2013; 2006 (2005): 79-80.

26. Ministerio de Educación. Buscador de carreras. mifuturo.cl. 